\title{
The Simultaneous Use of Positive Airway Pressure and Oral Appliance Therapy With and Without Connector: A Preliminary Study
}

\author{
Yasue Tanaka, $\mathrm{PhD}^{1,2}$; J. Michael Adame, DDS³; Adolfo Kaplan, MD; Fernanda R. Almeida, PhD' \\ ${ }^{1}$ Department Oral Health Sciences, Faculty of Dentistry, University of British Columbia, Vancouver, BC, Canada: ${ }^{2}$ Division of Aging \\ and Geriatric Dentistry, Tohoku University Graduate School of Dentistry, Sendai, Japan; ${ }^{3}$ Adame Dental Sleep Medicine, Edinburg, \\ Texas; ${ }^{4}$ Pulmonary and Sleep Center of the Valley, McAllen, Texas
}

\begin{abstract}
Study Objectives: Information on the efficacy of the combination therapy $(\mathrm{CT})$ of positive airway pressure (PAP) with oral appliance (OA), and the connection between PAP mask and OA in obstructive sleep apnea (OSA) is lacking. The goal of the study was to clarify the efficacy of CT with and without connector regarding self-reported side effects and sleepiness.

Methods: Twenty-eight patients with OSA who had previously experienced both solo-PAP and OA therapy were allocated to either CT of PAP and OA with connector (Connected-CT) or CT of PAP and OA without connector (Disconnected-CT). Participants reported the frequency of 17 different types of side effects, Epworth Sleepiness Scale (ESS), and short version of the Functional Outcomes of Sleep Questionnaire (FOSQ-10), both at baseline and at the 2-month follow-up. PAP data such as respiratory event index, PAP pressure, and mask leak were obtained from devices at $\mathrm{t}$ follow-up, and compared with those during solo-PAP therapy.
\end{abstract}

Results: Twelve participants in the Disconnected-CT group $(60.7 \pm 11.3$ years old) and 13 in the Connected-CT group (54.5 \pm 8.6 years old) completed the entire course of this study. Both Disconnected-CT and Connected-CT lowered the frequency of several types of PAPrelated side effects. ESS and FOSQ-10 were significantly improved after CT in both groups compared to baseline. There was no significant difference in respiratory event index, PAP pressure, or leaks between solo-PAP therapy and either CTs.

Conclusion: The use of PAP and OA simultaneously can improve self-reported side effects related to PAP therapy and sleepiness without change in PAP parameters.

Keywords: combination therapy, ESS, FOSQ-10, obstructive sleep apnea, oral appliance, positive airway pressure, side effects

Citation: Tanaka Y, Adame JM, Kaplan A, Almeida FR. The simultaneous use of positive airway pressure and oral appliance therapy with and without connector: A preliminary study. J Dent Sleep Med. 2022;9(1)

\section{INTRODUCTION}

Positive airway pressure (PAP) is considered to be the first-line treatment for obstructive sleep apnea (OSA). The best treatment outcomes are dose dependent, ${ }^{1}$ where the increasing hours of PAP use are related to an improvement in sleepiness, blood pressure, and other health outcomes. ${ }^{2,3}$ Therefore, improvements in adherence are necessary for patients who are intolerant of PAP to achieve maximum therapeutic effects from the therapy. However, nonadherence rate to PAP is as high as $34.1 \%{ }^{4}$ The factors affecting PAP adherence are categorized into four groups: sociodemographic characteristics, disease severity, psychosocial factors, and side effects. ${ }^{5}$ Several studies have shown that PAP-related side effects can associate with nonadherence to PAP. ${ }^{6-8}$ The common PAP-related side effects are intolerance of PAP pressure and mask interface, nasal problems, claustrophobia, difficulty initiating sleep, dry mouth, and unintentional removal of PAP mask during sleep. ${ }^{9}$ The reduction of these side effects might be a promising approach for achieving favorable PAP adherence.

However, an oral appliance (OA) that holds the mandible in the anterior position during sleep is considered to be the alternative to PAP, especially for the patients with mild to moderate $\mathrm{OSA}^{10}$ or for patients who are unable or unwilling to use PAP. ${ }^{11}$ Although PAP is considered to be more effective than an OA in reducing the apnea-hypopnea index (AHI), the OA also reduces AHI and shows higher hours per night used compared to PAP. ${ }^{12}$ However, the efficacy of OA therapy might vary depending on patient characteristics such as age, sex, and body mass index, and the success rate of OA therapy for patients with mild to moderate OSA ranges from $24 \%$ to $75 \%{ }^{13}$

Combination therapy (CT) of PAP and OA can be an alternative treatment for those who are intolerant to PAP and have incomplete response to OA. The recent study by Tong et al. showed that CT with OA and PAP could lower therapeutic PAP requirements and normalize pharyngeal pressure swings compared to PAP alone. ${ }^{14}$ Liu and colleagues showed that the simultaneous use of OA and PAP for patients with severe OSA lowered AHI, oxygen desaturation index, and PAP pressure compared to the sole use of OA therapy or PAP. ${ }^{15}$ The reduction of PAP pressure might contribute to improvement of adherence to PAP and therefore promote favorable health outcomes. However, Léotard and colleagues ${ }^{16}$ showed no improvement in adherence to PAP and worse self-reported pain with the combination of nasal mask and OA, compared to PAP with oronasal mask. Efficacy of the CT of PAP with OA is still 
unclear.

A PAP interface that connects the OA to PAP mask directly could allow a secure fit with the patient's face or head without any straps or bands and therefore could reduce the side effect related to the straps. TAP PAP CS (Airway Management Inc., Texas) is one such device already in the market. The nasal pillows of this device are anchored to the maxilla via dentition and expected to reduce discomfort of conventional PAP mask. However, the effects of the connection between OA and PAP mask have not been studied well.

The primary aim of this preliminary prospective study was to clarify the efficacy of CT with and without connector regarding self-reported side effects and sleepiness. The secondary aim was to clarify whether there were any differences in PAP data between solo-PAP and either CTs.

\section{MATERIALS AND METHODS}

\section{Participants}

Patients with OSA who had previously experienced both PAP and OA therapy independently of each other were recruited for this study. The inclusion criteria for participation were (1) age older than 18 years, (2) OSA with $\mathrm{AHI} \geq 5$ events per hour tested by polysomnography, (3) intolerance to PAP, and (4) incomplete response to OA therapy. Intolerance to PAP was determined by four questions according to self-reported side effects related to PAP use (high PAP, leak from mask, problems of exhaling, and uncomfortable pressure of mask). Patients were asked how often they experienced those four side effects and chose from five options; "never", "seldom", "sometimes", "often" or "very often'. Only those who answered "sometimes," "often," or "very often" at least once were included in this study. OAs were selected empirically by a dentist (MA) with sufficient clinical experience, based on the patient's clinical presentation, the author's clinical judgement, and in discussion with the patient. In each case, OA was titrated to the maximal forward position without pain or discomfort, and the amount of time in the titration period varied between 2 to 4 months. Incomplete response to OA therapy was defined as residual AHI $\geq 5$ events per hour tested by polysomnography or level III home sleep testing monitor (ApneaLink Air ${ }^{\mathrm{TM}}$, ResMed).

Exclusion criteria were (1) history of daily use of alcohol, (2) narcotics or central nervous system depressants, and (3) pregnancy. The written consent form was obtained from each participant. The protocol of this study was approved by the local ethics committee (Western Institutional Review Board; study number 1175023). This trial was registered with a National Institutes of Health clinical trial (NCT04029311).

\section{Interventions}

Participants were randomly assigned to either one of two CTs: CT with PAP connected to OA (Connected-CT) or CT with PAP disconnected from OA(Disconnected-CT). Participants who were assigned to Disconnected-CT were treated with PAP and OA, where PAP and OA were not connected but used simultaneously (Figure 1, a). Participants assigned to Connected-CT were treated with PAP and a custom-made nasal interface connected to an OA (Figure 1, b and c). In both groups, OAs used during CT were the same as those used during OA therapy alone and had already been titrated. In the Connected-CT group, there was a component that was secured to the OA usually via acrylic material and typically located just beyond the incisal edge of the anterior maxillary teeth. This component provides a direct connection between $\mathrm{OA}$ and interface. The OA was used as an anchor for an interface that connects to a nasal mask/pillows so that there is no need to use straps or bands, which are usually uncomfortable.

The participants used various PAP machine that had been prescribed by their sleep physicians, such as AirSense 10 AutoSet (ResMed, San Diego, CA), REMstar Auto AFlex (Koninklijke Philips N.V, Netherlands), ICON Auto (Fisher \& Paykel Healthcare, New Zealand), and BiPAP Pro Bi-Flex (Philips).

\section{Data Collection}

Follow-up visits were scheduled 1 and 2 months from the start of CT. Participants reported the frequency of 17 different types of side effects related to PAP therapy (Table 1) regarding previously experienced solo-PAP therapy at baseline, and regarding CT at the 2-month follow-up visit. As mentioned before, for each side effect, participants chose one from five options; "never", "seldom", "sometimes", "often" or "very often'. Participants completed the Epworth Sleepiness Scale ${ }^{17}$ (ESS) and a short version of the Functional Outcomes of Sleep Questionnaire $^{18}$ (FOSQ-10) at baseline and at the 2-month follow-up visit. Finally, participants were asked about likes and dislikes about the experienced therapies, i.e. OA therapy, solo-PAP therapy, and either of CTs.

Initially, 28 participants were included in this study, and randomly assigned into either Disconnected-CT $(n=15)$ or Connected-CT $(n=13)$. Three participants in the Disconnected-CT group dropped out of the study before follow-up. As a result, 25 participants (12 men and 13 women, mean age of $57.5 \pm 10.2$ ) completed the entire course of this study.

Fourteen participants (6 in the Disconnected-CT group and 8 in the Connected-CT group) who used a specific auto PAP machine (AirSense 10 AutoSet, ResMed) were included for PAP data analysis, because algorithms to provide pressure and detect events varied between the 


\section{Table 1. Side Effects Related to Positive Airway Pressure}

\section{Side effects}

High pressure of PAP

Air leak from mask

Problems of exhaling

Uncomfortable mask pressure

Blocked nose

Runny nose

Nosebleed

Dry throat

Irritated eyes

Irritated bowel

Transient deafness

Uncomfortable feeling due to PAP use

Increase of awakenings

Cold air

Disturbing noise

Anxiety

Unconscious need to remove in the night

PAP $=$ positive airway pressure.

types of devices. ${ }^{19}$ Respiratory event index which was referred to as "AHI" on the PAP device but sleep status was not considered, 95th percentile value of pressure, and 95th percentile value of leak for the past month were downloaded from PAP devices at follow-up visits. The PAP data during solo-PAP therapy were collected retrospectively from the patients' medical record. In case the data did not exist, participants were asked to undergo solo-PAP therapy for 1 week to 1 month in advance of CT. The average period between the day on which solo-PAP data were collected and the day of the follow-up visit was $133 \pm 115$ days.

\section{Statistical Analysis}

Data are shown as mean \pm standard deviations unless otherwise noted. Wilcoxon rank-sum test was used to

\section{Figure 1. Interface and oral appliance.}

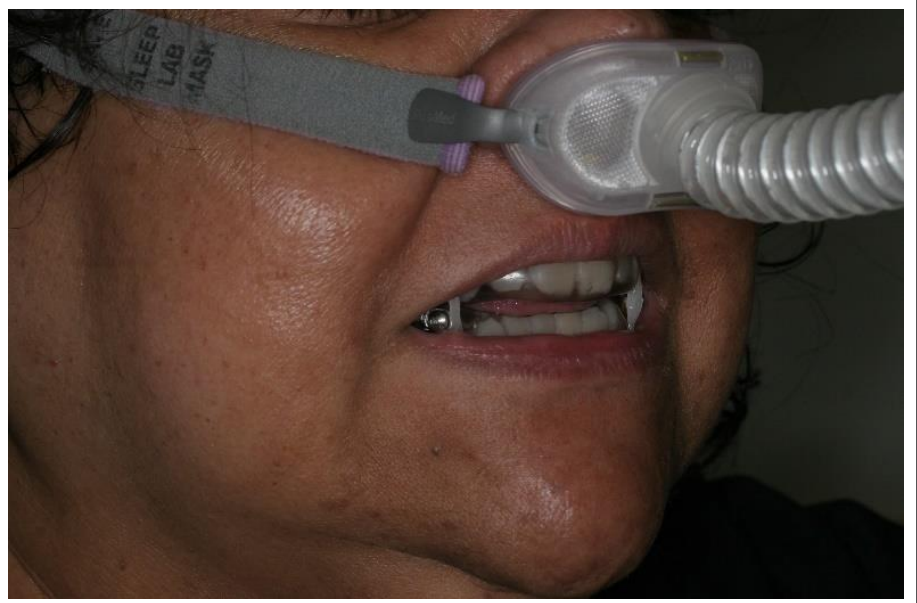

(a) Disconnected-combination therapy (CT); nasal mask and OA were disconnected from each other. The nasal mask was secured to the head by a strap.

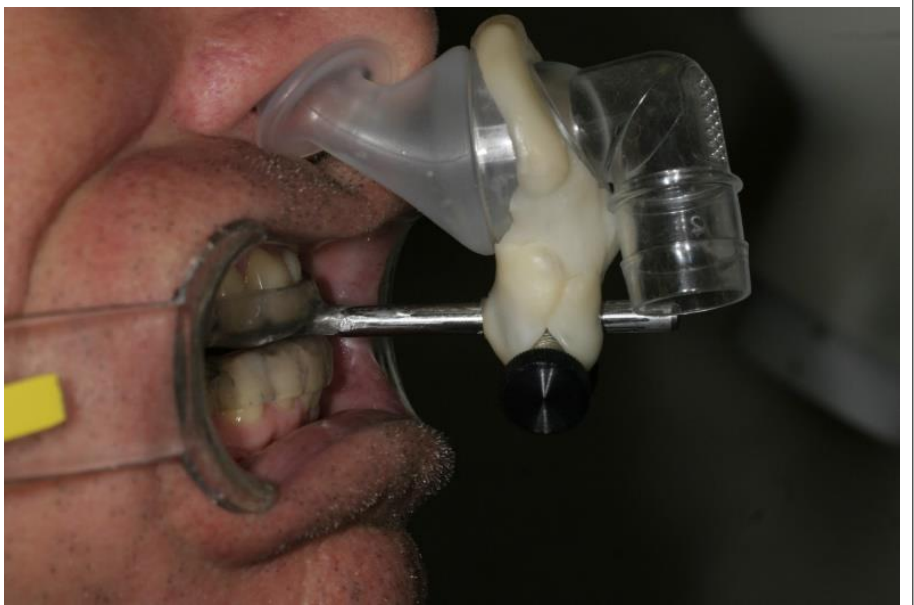

(b) Connected-CT; nasal mask and OA were connected to each other.

(c) Connected-CT; OA with connector out of the mouth.

compare the differences between the two groups at baseline. Wilcoxon signed-rank test was used for comparison between baseline and after $\mathrm{CT}$ and between 


\section{Table 2. Baseline Characteristics}

Total

\begin{tabular}{|c|c|c|c|c|c|c|c|}
\hline & & $\begin{array}{c}\text { Disconnected- } \\
\text { CT } \\
n=12\end{array}$ & $\begin{array}{c}\text { Connected } \\
\text {-CT } \\
n=13\end{array}$ & $\mathrm{p}$ & $\begin{array}{c}\text { Disconnected- } \\
\text { CT } \\
n=6\end{array}$ & $\begin{array}{c}\text { Connected } \\
\text {-CT } \\
\mathrm{n}=8\end{array}$ & $\mathrm{p}$ \\
\hline & Male, $n(\%)$ & $5(42)$ & $7(54)$ & & $4(67)$ & $5(62)$ & \\
\hline & Female, $n(\%)$ & $7(58)$ & $6(46)$ & & $2(33)$ & $3(38)$ & \\
\hline$B M I\left(k g / m^{2}\right)$ & & $34.8 \pm 9.1$ & $32.9 \pm 7.5$ & 0.35 & $32.1 \pm 2.9$ & $31.1 \pm 7.0$ & 0.30 \\
\hline \multirow[t]{3}{*}{$\begin{array}{l}\text { AHI } \\
\text { (events/h) }\end{array}$} & & $21.9 \pm 22.4$ & $31.6 \pm 27.3$ & 0.32 & $29.1 \pm 29.8$ & $29.7 \pm 19.3$ & 0.80 \\
\hline & $\begin{array}{l}5 \leq A H I<15, \quad n \\
(\%)\end{array}$ & $8(67)$ & $6(46)$ & & $4(67)$ & $3(38)$ & \\
\hline & $30 \leq A H I, n(\%)$ & $3(25)$ & $6(46)$ & & $2(33)$ & $4(50)$ & \\
\hline FOSQ-10 & & $30.1 \pm 7.9$ & $25.5 \pm 6.5$ & 0.13 & $30.5 \pm 9.0$ & $27.4 \pm 6.8$ & 0.43 \\
\hline$E S S$ & & $8.8 \pm 5.7$ & $12.2 \pm 4.8$ & 0.13 & $10.8 \pm 6.9$ & $11.8 \pm 5.7$ & 0.85 \\
\hline
\end{tabular}

Mean \pm SD. AHI = apnea-hypopnea index, BMI = body mass index, CT = combination therapy, ESS = Epworth

Sleepiness Scale, FOSQ-10 = short version of the Functional Outcomes of Sleep Questionnaire

Figure 2. Self-reported side effects related to positive airway pressure.

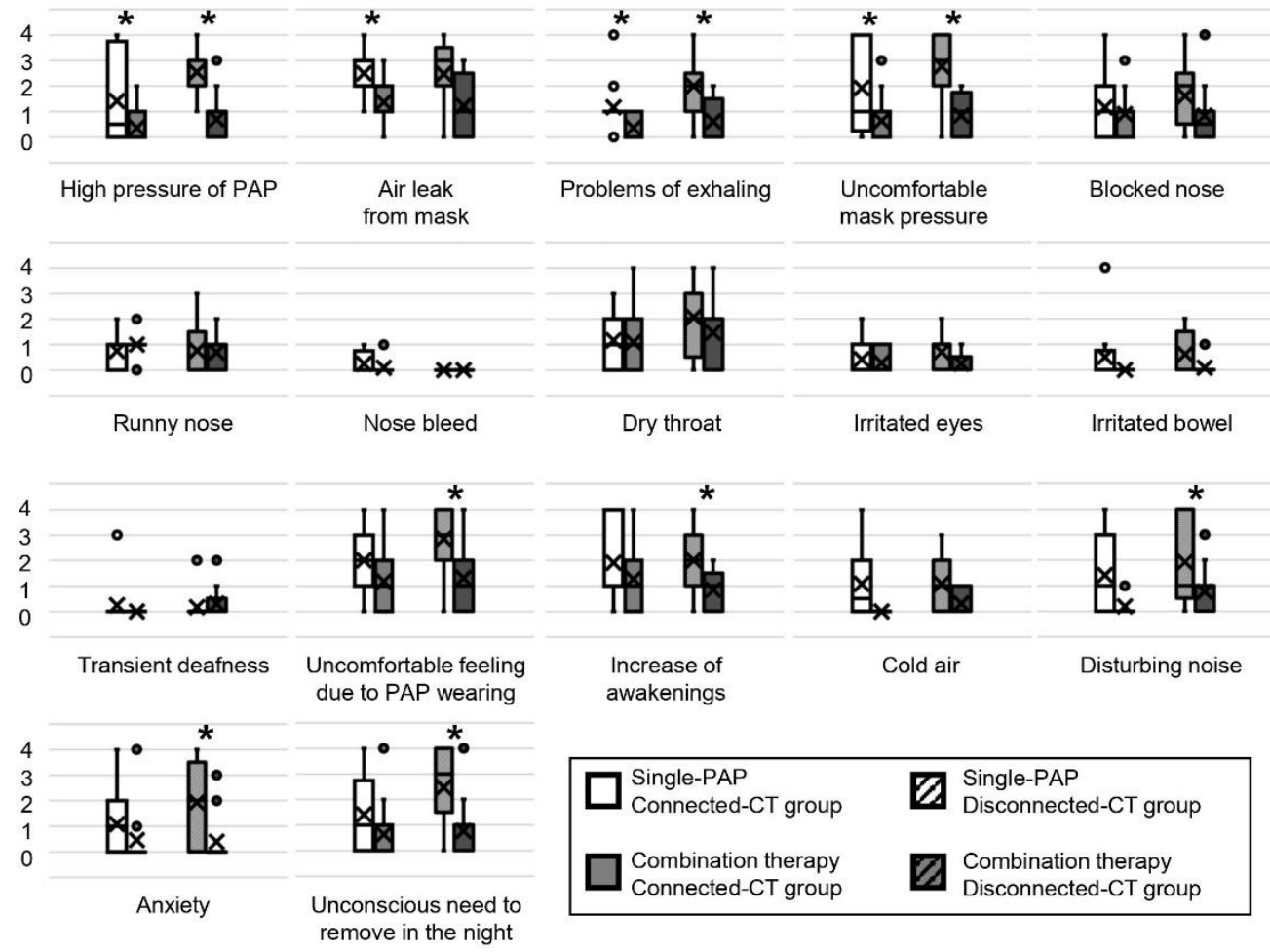

$0=$ never, 1 =seldom, $2=$ sometimes, $3=$ often, $4=$ very often

solo-PAP therapy and CT, within each group. Statistical significant difference was set at $P<0.05$. All statistical analyses were performed by JMP Pro 14 (SAS Institute Inc.) 


\section{RESULTS}

Baseline characteristics of 25 participants who completed the study are shown in Table 2. Although no significant differences were observed between the Disconnected-CT and Connected-CT groups, participants in the Connected-CT group tended to be younger $(P=0.10)$ and have lower FOSQ $(P=0.13)$ and higher ESS $(P=0.13)$, compared to the Disconnected-CT group.

Of the 25 participants, 16 (64\%) experienced high PAP pressure, $22(88 \%)$ experienced leak from mask, 11 (44\%) had problems exhaling, and 17 (68\%) experienced uncomfortable pressure of the mask more than "sometimes" at baseline. In the Disconnected-CT group, there were 6 Herbst ${ }^{\circledR}, 3$ TAP 3 Elite (Airway Management, Inc., Dallas, TX), 1 EMA ${ }^{\circledR}, 1$ Dorsal (DynaFlex, St. Ann, MO), and 1 SnoreHook appliances. In the Connected-CT group, there were 6 TAP 3 Elite, 5 Herbst ${ }^{\circledR}$, and 2 EMA ${ }^{\circledR}$ appliances. Residual AHI with OA alone ranged from 7.0 to 48.7 (15.6 $\pm 10.1)$.

The comparisons of side-effect frequency within each group were shown in Figure 2. "High pressure of PAP," "problem of exhaling," and "uncomfortable mask pressure" with both CT groups were significantly less frequent than during solo-PAP therapy. Only the Disconnected-CT group statistically decreased the frequency of "air leak." Only the Connected-CT group statistically decreased the frequency of side effects described as "uncomfortable feeling due to PAP device wearing," "increase of awakening," "disturbing noise," "anxiety," and "unconscious need to remove in the night". In both groups, participants tended to prefer CT to solo-PAP therapy (Figure 3).

Figure 3. Self-reported preference of each therapy.



$\mathrm{PAP}=$ positive airway pressure, $\mathrm{OA}=$ oral appliance, $\mathrm{CT}=$ combination therapy.

ESS was significantly decreased from baseline in both groups $(8.8 \pm 5.7$ vs. $5.5 \pm 4.0, P=0.011$ for the
Disconnected-CT group; $12.2 \pm 4.8$ vs. $5.6 \pm 2.7, P=0.0003$, for the Connected-CT group; Figure 4, a). FOSQ-10 score was significantly increased in both groups $(30.1 \pm 7.9$ vs. $36.3 \pm 3.8, P=0.020$, for the Disconnected-CT group; 25.5 \pm 6.5 vs. $36.2 \pm 2.8, P<0.0001$, for the Connected-CT group; Figure 4, b).

Baseline characteristic of the participants who were included in the PAP data analysis are shown in Table 2. No significant difference was observed between both groups. The mean usage duration was $6.7 \pm 1.7$ hours for the Disconnected-CT group and $5.5 \pm 2.6$ hours for the Connected-CT group. There was no significant difference between solo-PAP therapy and $\mathrm{CT}$, in respiratory event index ( $3.8 \pm 2.3$ vs. $5.7 \pm 3.7, P=0.84$, for the DisconnectedCT group; $5.2 \pm 2.8$ vs. $3.8 \pm 3.0, P=0.31$, for the ConnectedCT group), therapeutic pressure $(9.5 \pm 1.9$ vs. $9.5 \pm 1.7$, $P=0.75$, for the Disconnected-CT group; $10.6 \pm 2.2$ vs. $9.3 \pm 1.7, P=0.30$, for Connected-CT group), and amount of leak $(20.7 \pm 6.4$ vs. $19.1 \pm 5.4, P=0.43$, for the DisconnectedCT group; $21.1 \pm 7.2$ vs. $17.8 \pm 12.8, P=0.40$, for the Connected-CT group).

\section{DISCUSSION}

In this preliminary study, both Disconnected-CT and Connected-CT improved ESS and FOSQ-10. The frequency of self-reported side effects was decreased by both CTs; nevertheless, PAP data did not show any significant difference between solo-PAP therapy and either CT. Moreover, participants tended to prefer the CTs to soloPAP therapy. At the end of this trial, 25 of 28 patients were able to use the proposed CT rather than no therapy. Because subjective liking of the therapy would have a great effect on long-term adherence to PAP, both CTs could contribute to favorable health outcomes, even if there is no change in PAP parameters.

Connected-CT significantly reduced the frequency of more types of side effects than Disconnected-CT. For some side effects, Disconnected-CT also reduced the frequency, though the reductions were not statistically significant. The reduction of side effects in this study could contribute to the long-term adherence to PAP and other favorable outcomes such as reduction of daytime sleepiness. The 12month prospective study, which investigated 275 patients with OSA and cardiovascular disease, showed that the predictors of the 1-year PAP adherence were the side effect and PAP use at 1 month. ${ }^{7}$ Similarly, Borel and colleagues ${ }^{8}$ showed that PAP-related side effects as well as type of mask were associated with PAP nonadherence. In the recent study by Rotty et al. ${ }^{6}$, multivariable logistic regression analyses showed that mask-related side effects are independently associated with PAP nonadherence and positively associated with ESS score in patients treated over the long term with PAP. Considering these literature findings and study results, the reduction of the PAP-related 
Figure 4: Epworth Sleepiness Scale and Functional Outcomes of Sleep Questionnaire-10 score at baseline and follow-up.
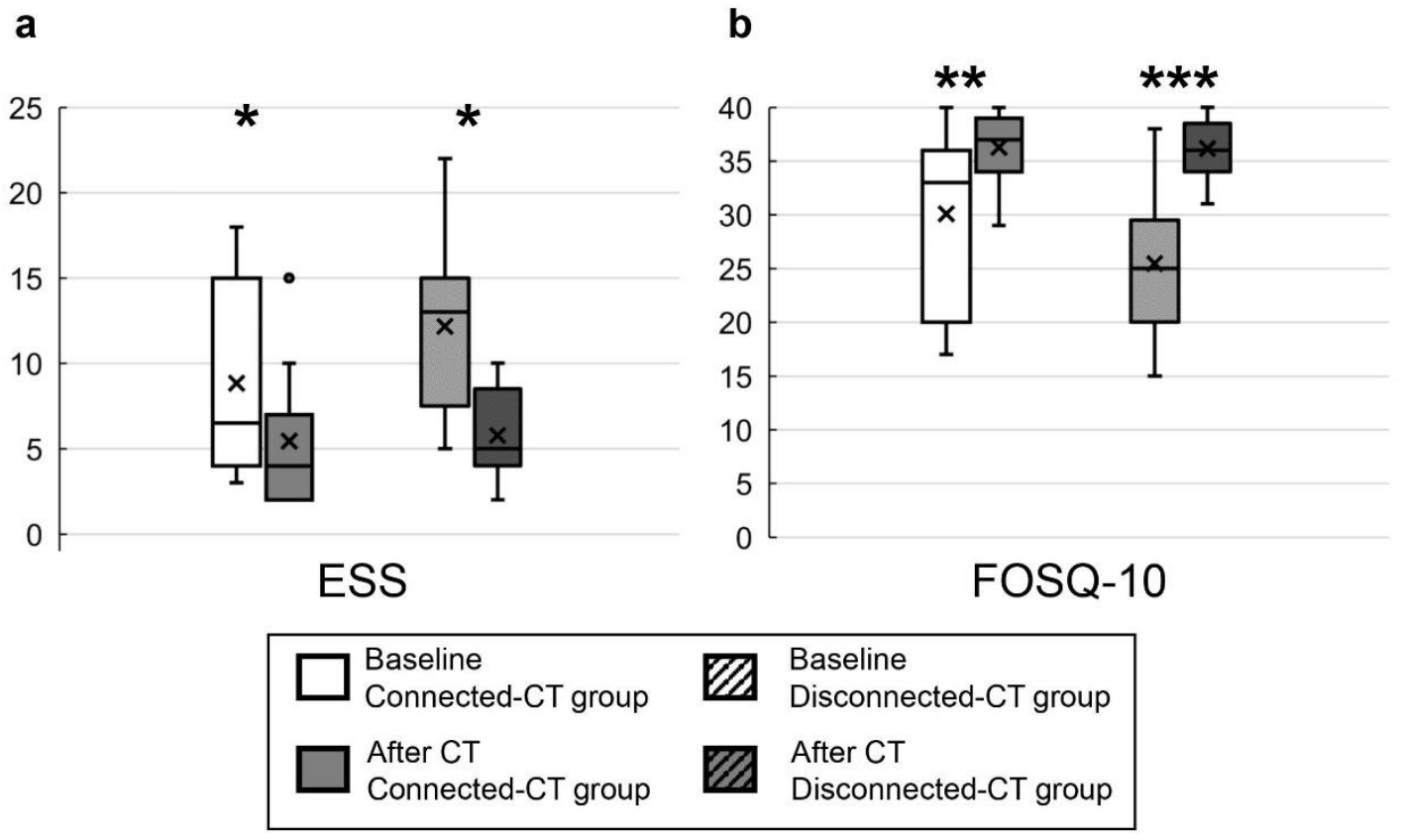

side effects by using OA and PAP simultaneously could improve the long-term adherence to PAP. In the future, long-term follow-up would show the efficacy of these CTs on PAP adherence and other health outcomes.

The results of this study showed no statistical difference in PAP pressure between solo-PAP and CT, which is inconsistent with the previous studies ${ }^{15,20}$ that showed the therapeutic pressure on CT with PAP and OA was significantly lower than that on solo-PAP therapy. However, when looking at data individually, for 1 of 6 participants in the Disconnected CT group and 4 of 8 participants in the Connected CT group, PAP pressure during CT was more than $1 \mathrm{cmH}_{2} \mathrm{O}$ lower than during soloPAP therapy. It is supposed that pressure during CT could only be lowered in patients with some factors. One possible factor is the severity of OSA. In the study by Liu et al, ${ }^{15}$ all participants were patients with severe OSA $(\mathrm{AHI} \geq 30)$ and the therapeutic pressure during solo-PAP therapy was $\geq 15$ $\mathrm{cmH}_{2} \mathrm{O}$, and had lower pressure on CT than on solo PAP. The other possible factor is self-reported high pressure during solo-PAP therapy. All participants in the study by El-Solh et a ${ }^{20}$ were intolerant to solo PAP therapy because of high pressure based on patient report and all but one participants achieved lower pressure on CT. In the current study, the sample size was too small to assess these factors. Future research with a large sample size is needed to clarify the predictors for achieving lower pressure on CT.

Despite the fact that there was no difference in pressure or leak between solo PAP therapy and either CT, participants described that the side effects during CT regarding high pressure and leaks were less frequent than during solo PAP therapy. This finding is consistent with the results from Rotty et al. ${ }^{6}$ In their study, patient-reported leaks were not associated with device-reported leaks. They considered that not only the strength of the leak flow but also how it feels on a patient's skin/eyes and associated noises could be perceived as a leak by patients, whereas the PAP machine only detected leak flows.

There are a couple of studies ${ }^{21,22}$ that showed the efficacy of CT of PAP and OA with connector. Similar to our results, De Vries et al. ${ }^{21}$ showed that participants had fewer PAP-related complaints with the hybrid therapy (combining PAP with OA by means of a connection unit) than with solo PAP therapy. Prehn and Swick ${ }^{22}$ retrospectively reviewed the charts of 75 patients who underwent $\mathrm{CT}$ with the TAP PAP custom facemask (custom facemask connected OA). They concluded that patients in whom solo PAP therapy failed would benefit the most from the CT with the TAP PAP custom facemask. In addition to the TAP PAP system, we also utilized similarly anchored interfaces that were developed by one of the authors (MA), but not yet reported by any other group.

Because our study was implemented in the general clinical setting, there were several limitations. First, a relatively small sample size made it difficult to generalize the findings, especially for PAP data analysis in which only those who were using a specific PAP device were included. Second, the order and the duration of solo PAP and OA 
treatment that each participant received before they participated in this study varied. This study was conducted as a traditional 'real life' clinical setting such that once the study inception date was determined, those who met the inclusion criteria were invited to the study and as such, their backgrounds on timing for diagnosis and first line of treatments were variable. Third, only the data recorded by the PAP device were used to compare the respiratory parameters during solo PAP therapy with CTs. Because pulse oximetry was performed during CT but not during solo PAP therapy, we excluded it from the analysis. Last, due to the study design, the direct comparison between Disconnected-CT and Connected-CT was difficult. To gain a deeper understanding of the differences between both CTs, a crossover study with larger sample size would be necessary.

In conclusion, the CT of PAP and OA both with and without connector improved self-reported side effects related to PAP and sleepiness without changing PAP parameters in this small preliminary study.

\section{ABBREVIATIONS}

AHI; apnea-hypopnea index

PAP; positive airway pressure

$\mathrm{CT}$; combination therapy

ESS; Epworth Sleepiness Scale

FOSQ-10; short version of the Functional Outcomes of

Sleep Questionnaire

OA; oral appliance

OSA; obstructive sleep apnea

PSG; polysomnography

\section{ACKNOWLEDGEMENTS}

The authors would like to thank Ricardo Abreu, MD, Juan M. Ortiz, MD, Mario Gomez, MD, Joseph Penn, MD, Alfredo R. Aruco Brown, MD, and Margie Rodriguez, FNP-C for assistance with project design, and Tony Abreu, BS, RPSGT, Aspin Tobola, CDA, and Juan Carlos Alaniz, CDA for assistance with conducting the trial.

\section{REFERENCES}

1. Weaver TE, Maislin G, Dinges DF, et al. Relationship between hours of CPAP use and achieving normal levels of sleepiness and daily functioning. Sleep. 2007; 30: 711-719.

2. Martínez-García MA, Capote F, Campos-Rodríguez F, et al. Effect of $\mathrm{CPAP}$ on blood pressure in patients with obstructive sleep apnea and resistant hypertension: the HIPARCO randomized clinical trial. JAMA. 2013;310(22):2407-2415.

3. Zimmerman ME, Arnedt JT, Stanchina M, Millman RP, Aloia MS Normalization of memory performance and positive airway pressure adherence in memory-impaired patients with obstructive sleep apnea. Chest. 2006; 130(6):1772-1778.

4. Rotenberg BW, Murariu D, Pang KP. Trends in CPAP adherence over twenty years of data collection: a flattened curve. J Otolaryngol Head Neck Surg. 2016;45(1):43.
5. Mehrtash M, Bakker JP, Ayas N. Predictors of continuous positive airway pressure adherence in patients with obstructive sleep apnea. Lung. 2019;197(2):115-121.

6. Rotty MC, Suehs CM, Mallet JP, et al. Mask side-effects in long-term CPAP-patients impact adherence and sleepiness: the InterfaceVent real-life study. Respir Res. 2021;22(1):17.

7. Chai-Coetzer CL, Luo YM, Antic NA, et al. Predictors of long-term adherence to continuous positive airway pressure therapy in patients with obstructive sleep apnea and cardiovascular disease in the SAVE study. Sleep. 2013;36(12):1929-1937.

8. Borel JC, Tamisier R, Dias-Domingos S, et al. Type of mask may impact on continuous positive airway pressure adherence in apneic patients. PLoS One. 2013;8(5):e64382

9. Vanderveken OM, Hoekema A. How to treat patients that do not tolerate continuous positive airway pressure. Breathe. 2010;7:157167.

10. Doff MH, Hoekema A, Wijkstra PJ, et al. Oral Appliance versus continuous positive airway pressure in obstructive sleep apnea Syndrome: a 2-year follow-up. Sleep. 2013;36(9):1289-1296.

11. Li W, Xiao L, Hu J. The comparison of CPAP and oral appliances in treatment of patients with OSA: a systematic review and metaanalysis. Respir Care. 2013;58(7):1184-95.

12. Sutherland K, Vanderveken OM, Tsuda H, et al. Oral appliance treatment for obstructive sleep apnea: an update. J Clin Sleep Med. 2014;10(2):215-227

13. Fukuda T, Tsuiki S, Kobayashi M, Nakayama H, Inoue Y. Selection of response criteria affects the success rate of oral appliance treatment for obstructive sleep apnea. Sleep Med. 2014;15(3):367-370.

14. Tong BK, Tran C, Ricciardiello A, et al. CPAP combined with oral appliance therapy reduces CPAP requirements and pharyngeal pressure swings in obstructive sleep apnea. J Appl Physiol (1985). 2020. Epub ahead of print.

15. Liu HW, Chen YJ, Lai YC, et al. Combining MAD and CPAP as an effective strategy for treating patients with severe sleep apnea intolerant to high-pressure PAP and unresponsive to MAD. PLoS One. 2018;13(4): $\mathrm{e} 0196319$.

16. Léotard A, Lesgoirres M, Daabek N, et al. Adherence to CPAP with a nasal mask combined with mandibular advancement device versus an oronasal mask: a randomized crossover trial. Sleep Breath. 2019;23(3):885-888.

17. Johns MW. A new method for measuring daytime sleepiness: the Epworth sleepiness scale. Sleep. 1991;14(6):540-545.

18. Chasens ER, Ratcliffe SJ, Weaver TE. Development of the FOSQ-10: a short version of the Functional Outcomes of Sleep Questionnaire. Sleep. 2009;32(7):915-919.

19. Johnson KG, Johnson DC. Treatment of sleep-disordered breathing with positive airway pressure devices: technology update. Med Devices (Auckl). 2015;8:425-437.

20. El-Solh AA, Moitheennazima B, Akinnusi ME, Churder PM, Lafornara AM. Combined oral appliance and positive airway pressure therapy for obstructive sleep apnea: a pilot study. Sleep Breath. 2011;15(2):203-208

21. de Vries GE, Doff MH, Hoekema A, Kerstjens HA, Wijkstra PJ. Continuous positive airway pressure and oral appliance hybrid therapy in obstructive sleep apnea: patient comfort, compliance, and preference: a pilot study. JDSM. 2016;3(1):5-10.

22. Prehn RS, Swick T. A descriptive report of combination therapy (custom face mask for CPAP integrated with a mandibular advancement splint) for long-term treatment of OSA with literature review. JDSM. 2017;4(2):29-36. 


\section{SUBMISSION \& CORRESPONDENCE INFORMATION}

Submitted November 2, 2020

Submitted in final revised form March 31, 2021

Accepted for publication September 17, 2021

Address correspondence to: J. Michael Adame, DDS, 206 W. Mahl, Edinburg, TX. Email: jma@jmichaeladamedds.com

\section{DISCLOSURE STATEMENT}

The authors report no conflicts of interest. 